Marquette University

e-Publications@Marquette

8-2004

\title{
Spectroscopic and X-ray Crystallographic Characterization of Bestatin Bound to the Aminopeptidase from Aeromonas (Vibrio) proteolytica
}

\author{
Carin C. Stamper \\ Brandeis University \\ David L. Bienvenue \\ Utah State University \\ Brian Bennett \\ Marquette University, brian.bennett@marquette.edu \\ Dagmar Ringe \\ Brandeis University \\ Gregory A. Petsko \\ Brandeis University
}

See next page for additional authors

Follow this and additional works at: https://epublications.marquette.edu/physics_fac

Part of the Physics Commons

\section{Recommended Citation}

Stamper, Carin C.; Bienvenue, David L.; Bennett, Brian; Ringe, Dagmar; Petsko, Gregory A.; and Holz, Richard C., "Spectroscopic and X-ray Crystallographic Characterization of Bestatin Bound to the Aminopeptidase from Aeromonas (Vibrio) proteolytica" (2004). Physics Faculty Research and Publications. 38.

https://epublications.marquette.edu/physics_fac/38 


\section{Authors}

Carin C. Stamper, David L. Bienvenue, Brian Bennett, Dagmar Ringe, Gregory A. Petsko, and Richard C. Holz 
Marquette University

\section{e-Publications@Marquette}

\section{Physics Faculty Research and Publications/College of Arts and Sciences}

This paper is NOT THE PUBLISHED VERSION; but the author's final, peer-reviewed manuscript. The published version may be accessed by following the link in the citation below.

Biochemistry, Vol. 43, No. 30 (1 August 2004): 9620-9628. DOI. This article is (C) American Chemical Society Publications and permission has been granted for this version to appear in $\underline{\mathrm{e}}-$ Publications@Marquette. American Chemical Society Publications does not grant permission for this article to be further copied/distributed or hosted elsewhere without the express permission from American Chemical Society Publications.

\section{Spectroscopic and X-ray Crystallographic Characterization of Bestatin Bound to the Aminopeptidase from Aeromonas (Vibrio) proteolytica}

\section{Carin C. Stamper}

Program in Biophysics and Structural Biology, Departments of Biochemistry and Chemistry and the Rosenstiel Basic Medical Sciences Research Center, Brandeis University, Waltham, Massachusetts

David L. Bienvenue Department of Chemistry and Biochemistry, Utah State University, Logan, Utah Brian Bennett

National Biomedical EPR Center, Department of Biophysics, Medical College of Wisconsin, Milwaukee, Wisconsin

Dagmar Ringe

Program in Biophysics and Structural Biology, Departments of Biochemistry and Chemistry and the Rosenstiel Basic Medical Sciences Research Center, Brandeis University, Waltham, Massachusetts 


\section{Gregory A. Petsko}

Program in Biophysics and Structural Biology, Departments of Biochemistry and Chemistry and the Rosenstiel Basic Medical Sciences Research Center, Brandeis University, Waltham, Massachusetts

\section{Richard C. Holz}

Department of Chemistry and Biochemistry, Utah State University, Logan, Utah

\section{SUBJECTS:}

Peptides and proteins, Metals, Amines, lons

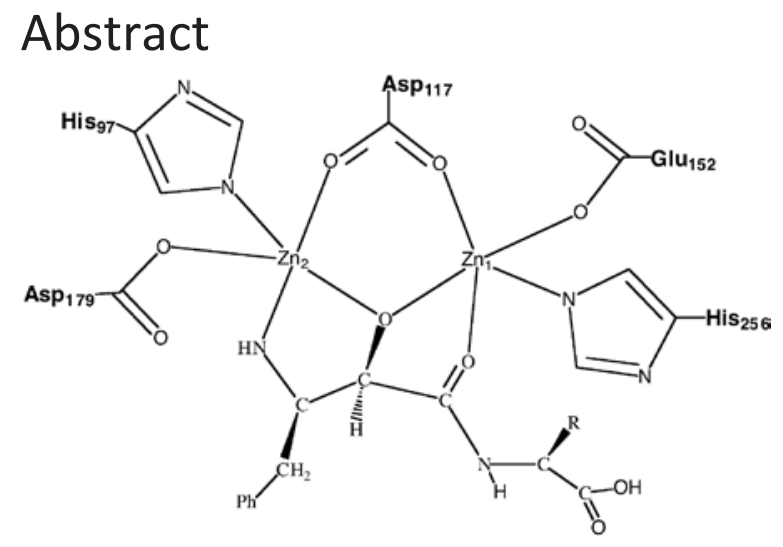

Binding of the competitive, slow-binding inhibitor bestatin ([(2S,3R)-3-amino-2-hydroxy-4-phenylbutanoy]leucine) to the aminopeptidase from Aeromonas proteolytica (AAP) was examined by both spectroscopic and crystallographic methods. Electronic absorption spectra of the catalytically competent [Co_(AAP)], [CoCo(AAP)], and [ZnCo(AAP)] enzymes recorded in the presence of bestatin revealed that both of the divalent metal ions in AAP are involved in binding bestatin. The electron paramagnetic resonance (EPR) spectrum of the [CoCo(AAP)]-bestatin complex exhibited no observable perpendicular- or parallel-mode signal. These data indicate that the two Co" ions in AAP are antiferromagnetically coupled yielding an $S=0$ ground state and suggest that a single oxygen atom bridges between the two divalent metal ions. The EPR data obtained for [CoZn(AAP)] and [ZnCo(AAP)] confirm that bestatin interacts with both metal ions. The X-ray crystal structure of the [ZnZn(AAP)]-bestatin complex was solved to $2.0 \AA$ resolution. Both side chains of bestatin occupy a welldefined hydrophobic pocket that is adjacent to the dinuclear $\mathrm{Zn}$ " active site. The amino acid residues ligated to the dizinc(II) cluster in AAP are identical to those in the native structure with only minor perturbations in bond length. The alkoxide oxygen of bestatin bridges between the two $\mathrm{Zn}^{\prime \prime}$ ions in the active site, displacing the bridging water molecule observed in the native [ZnZn(AAP)] structure. The $M-M$ distances observed in the AAP-bestatin complex and native AAP are identical (3.5 $\AA$ ) with alkoxide oxygen atom distances of 2.1 and $1.9 \AA$ from Zn1 and Zn2, respectively. Interestingly, the backbone carbonyl oxygen atom of bestatin is coordinated to $\mathrm{Znl}$ at a distance of $2.3 \AA$. In addition, the $\mathrm{NH}_{2}$ group of bestatin, which mimics the $\mathrm{N}$-terminal amine group of an incoming peptide, binds to Zn2 with a bond distance of $2.3 \AA$. A combination of the spectroscopic and X-ray crystallographic data presented herein with the previously reported mechanistic data for AAP has provided additional insight into the substrate-binding step of peptide hydrolysis as well as insight into important small molecule features for inhibitor design.

Aminopeptidases catalyze the hydrolysis of a wide variety of $\mathrm{N}$-terminal amino acids from proteins and polypeptide chains (1-3). Most enzymes in this group have broad substrate specificities and are widely 
distributed in both plant and animal tissues (2). Their biological and medicinal significance is extensive because of their many roles in the degradation of proteins and biologically active peptides, including hormones. The importance of understanding the reaction mechanism of aminopeptidases is underscored by their central role in several disease states including stroke, diabetes, cancer, HIV, bacterial infections, and neuropsychiatric disorders associated with the dysregulation of glutamatergic neurotransmission, such as schizophrenia, seizure disorders, and amyotrophic lateral sclerosis (ALS) (4-6). Recently, it has been shown that several naturally occurring hydroxyethyl isostere dipeptide metalloaminopeptidase inhibitors (i.e., bestatin, leuhistin, and actinonin) inhibit matrix degradation and invasion of extracellular matrixes by fibrosarcoma cells as well as decrease HIV viral load $(6,7)$. For these reasons, several metallopeptidases have become the subject of intense efforts in inhibitor design (8-13).

Aminopeptidases that have dimetallic active sites can be split into two distinct groups based on their active site structures (8). The first group includes the leucine aminopeptidases from bovine lens (bILAP), ${ }^{1}$ porcine kidney, tomato, and Escherichia coli (PepA) (Figure 1), while the second group contains the leucine aminopeptidases from Aeromonas (Vibrio) proteolytica (AAP) (14) and Streptomyces griseus (SAP) (Figure 1A) (15). AAP, while not a specific pharmaceutical target at this time, contains a dinuclear active site that is superimposable on the vast majority of dinuclear metallopeptidases that are potential pharmaceutical targets such as SAP, the daminopeptidase from Bacillus subtilis (DppA), the dapE-encoded $\mathrm{N}$-succinyl-I,I-diaminopimelic acid desuccinylase (DapE), the $\arg$-encoded $\mathrm{N}$-acetyl-I-ornithine deacetylase (ArgE), the carboxypeptidase $\mathrm{G}_{2}$ from Pseudomonas sp. strain RS-16 ( $\left.\mathrm{CPG}_{2}\right)$, and glutamate carboxypeptidase II (GCP-II), sometimes referred to as $N$-acetylated- $\alpha$-linked-acidic dipeptidase (NAALADase) $(8,14-17$. AAP has been structurally characterized to $1.8 \AA \AA$ resolution (1AMP) (18), but recently, its structure was determined to $1.2 \AA$ resolution $(19,20)$. In both structures, AAP was shown to contain a single globular domain with a centrally located mixed $\beta$ sheet sandwiched between $\alpha$ helices. The cocatalytic active site contains a ( $\mu$-aquo)( $\mu$-carboxylato)dizinc(II) core with a terminal carboxylate and histidine residue at each metal ion resulting in symmetric coordination spheres for the two active site $\mathrm{Zn}$ " ions (Figure 1B). Both $\mathrm{Zn}$ " ions reside in distorted tetrahedral coordination geometries with a Zn-Zn distance of $3.5 \AA$. A glutamate residue, Glu151, forms a hydrogen bond with the bridging water molecule, while the second oxygen atom is $3.4 \AA$ from the $\mathrm{N}^{\mathrm{e}}$ of His97, which is a ligand to Zn1. 
$\mathbf{A}$
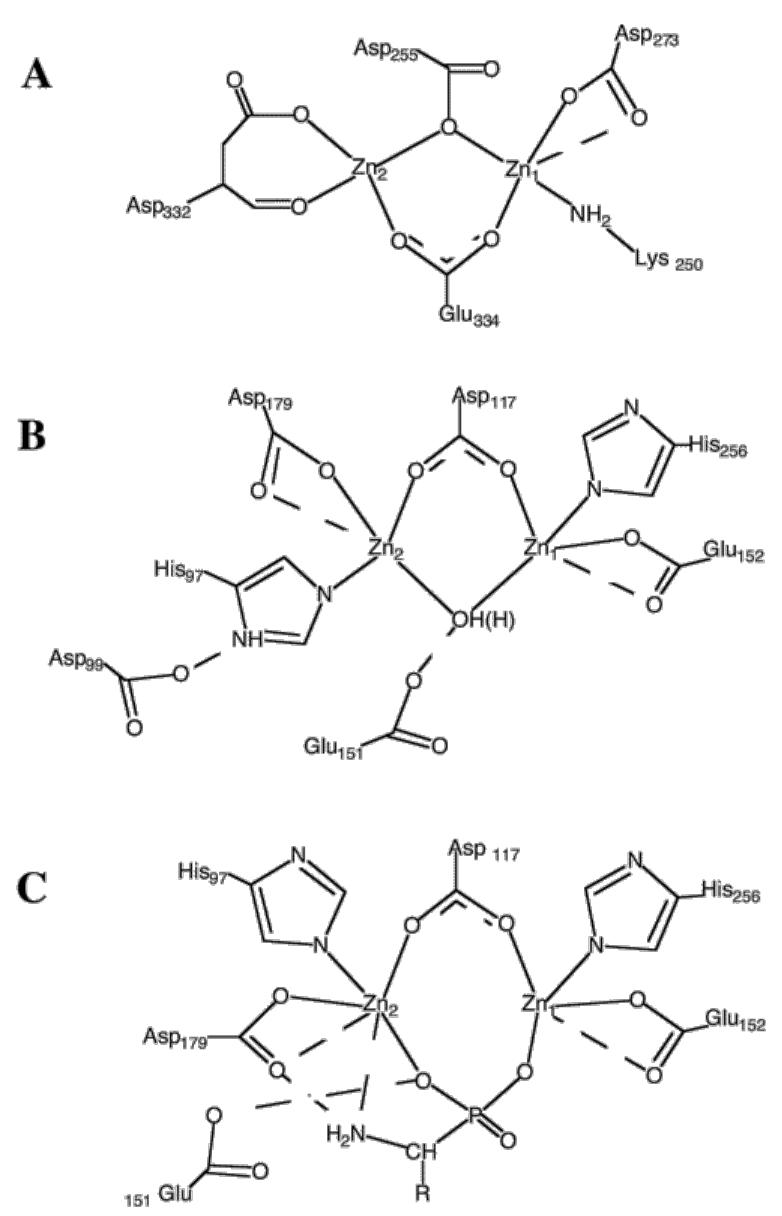

Figure 1 Schematic representations of the bILAP and AAP active sites and the AAP-LPA complex.

Aminopeptidase inhibitors with boronic acids (21-23), chloromethyl ketones (24), phosphonic acids (25-27), hydroxamate (28-30), and $\alpha$-hydroxyamide (24) functional groups have been previously studied. Peptide analogue aminopeptidase inhibitors of microbiological origin have not been examined in detail. One of these, bestatin ([(2S,3R)-3-amino-2-hydroxy-4-phenylbutanoy]-leucine) is a naturally occurring dipeptide isolated from cultures of Streptomyces olivoreticuli (31). Bestatin has been shown to be a competitive, slow-binding inhibitor of both bILAP and AAP with $K_{\mathrm{i}}^{*}$ values of 1.3 and $18 \mathrm{nM}$, respectively (32-34). For all known aminopeptidases, Xray crystallographic data of a bestatin-bound analogue has only been reported for bILAP (35). The X-ray crystal structure of the [ZnZn(bILAP)]-bestatin complex revealed that the N-terminal amino group coordinates to $\mathrm{Zn2}$, while the alkoxide moiety bridges between the two $\mathrm{Zn}^{\prime \prime}$ ions in the bILAP active site. Interestingly, the backbone carbonyl oxygen of bestatin is not bound to the dinuclear metal center in bILAP but is, instead, hydrogenbonded to the positively charged terminal amine of an active site lysine residue.

In an effort to gain insight into the structure of AAP in the Michaelis complex, we have studied the binding of the competitive, slow-binding inhibitor bestatin ([(2S,3R)-3-amino-2-hydroxy-4-phenylbutanoy]-leucine) to AAP using both spectroscopic and crystallographic methods. The spectroscopic and X-ray crystallographic data presented herein combined with the previously reported mechanistic data for AAP (14) provide additional insight into the substrate-binding step of peptide hydrolysis and important features for inhibitor design. 


\section{Materials and Methods}

\section{Enzyme Purification.}

All chemicals used in this study were purchased commercially and were of the highest quality available. The aminopeptidase from Aeromonas(Vibrio) proteolytica was purified from a stock culture kindly provided by Professor Céline Schalk. Cultures were grown according to the previously published procedure (36) with minor modifications (37) to the growth media. Purified enzyme was stored at $77 \mathrm{~K}$ until needed.

\section{Spectrophotometric Assay.}

AAP activity was measured by the method of Prescott and Wilkes (36) as modified by Baker et al. (22). In this assay, the hydrolysis of $0.5 \mathrm{mM}$ I-leucine $p$-nitroanilide (I-pNA) (10 mM Tricine at pH 8.0) was measured spectrophotometrically at $25^{\circ} \mathrm{C}$ by monitoring the formation of $p$-nitroaniline. The extent of hydrolysis was calculated by monitoring the increase in absorbance at $405 \mathrm{~nm}\left(\Delta \varepsilon_{405}\right.$ value of $p$-nitroaniline of $\left.10800 \mathrm{M}^{-1} \mathrm{~cm}^{-1}\right)$. One unit is defined as the amount of enzyme that releases $1 \mu \mathrm{mol}$ of $p$-nitroaniline at $25^{\circ} \mathrm{C}$ in $60 \mathrm{~s}$. Depletion of enzyme- bound zinc or cobalt was prevented by the addition of $0.1 \mathrm{mM} \mathrm{ZnSO}_{4}$ or $\mathrm{CoCl}_{2}$ to the buffer. The specific activity of purified Zn"-bound AAP was typically found to be 120 units per $\mathrm{mg}$ of enzyme. This value is identical to that reported by Prescott and Wilkes (36). Enzyme concentrations were determined from the absorbance at $280 \mathrm{~nm}$ with the value $\varepsilon_{280}=41800 \mathrm{M}^{-1} \mathrm{~cm}^{-1}(38)$. The accuracy of this value was checked by the Edelhoch method (39-41) using a 5:13:2 molar ratio mixture of $N$-acetyl-I-tryptophanamide/Gly-Tyr-amide/Icysteine to model AAP. The molar absorptivity determined from this method, $\varepsilon_{280}=43950 \mathrm{M}^{-1} \mathrm{~cm}^{-1}$ for AAP solubilized in $6 \mathrm{M}$ guanidine hydrochloride, was in excellent agreement with the previously reported value by Prescott et al. (38).

\section{Electronic Absorption and Electron Paramagnetic Resonance (EPR) Samples.}

[CoZn(AAP)] and [ZnCo(AAP)] were prepared by dialysis for $72 \mathrm{~h}$ at $4{ }^{\circ} \mathrm{C}$ against $10 \mathrm{mM}$ 1,10-phenanthroline monohydrochloride (o-phen) in $50 \mathrm{mM}$ Hepes buffer at pH 7.5 (42). AAP was then exhaustively dialyzed against metal-free (chelexed) $50 \mathrm{mM}$ HEPES buffer at $\mathrm{pH}$ 7.5. Any remaining metal ions were estimated by comparing the activity of the apo enzyme with a sample that had been reconstituted with $\mathrm{Zn}$ ". AAP incubated with $o$-phen typically had less than $5 \%$ residual activity after dialysis. Enzyme concentrations for UV-Vis and EPR samples were typically 1-2 mM. All buffers contained 20\% 2-propanol to prevent aggregation at high protein concentrations $(43,44)$. Metal insertion was effected by the direct addition, with efficient mixing, of 1 equiv of $\mathrm{MCl}_{2}$ (where $\mathrm{M}=\mathrm{Co}$ or $\mathrm{Zn}$; $\geq 99.999 \% \mathrm{CoCl}_{2}$, Strem Chemicals, Newburyport, Massachusetts; $99.999 \% \mathrm{ZnCl}_{2}$, Aldrich) followed by an incubation period for $30 \mathrm{~min}$ at $20-25^{\circ} \mathrm{C}$. The second metal was then inserted in the same manner, and the electronic absorption spectrum was recorded prior to freezing in liquid nitrogen for EPR spectroscopy. A 5-fold excess of bestatin was introduced onto the inside side wall of an EPR tube, and the enzyme sample was introduced above this as a plug of $\sim 2 \mathrm{~cm}$ in length. As earlier work has demonstrated by optical methods (45), violently flicking the above system facilitates rapid and efficient mixing of the reagents and rapid freezing was achieved by plunging the tube into a beaker of a mixture of liquid and solid methanol over liquid nitrogen. These same EPR samples were then used to record electronic absorption spectra of the AAP-bestatin complex.

\section{Spectroscopic Measurements.}

All spectrophotometric measurements were performed on a Shimadzu UV-3101PC spectrophotometer equipped with a constant temperature holder and a Haake (Type 423) constant-temperature circulating bath. The use of 200- $\mu \mathrm{L}, 1-\mathrm{cm}$ path-length microcuvettes (QS, Hellma) stoppered with rubber septa facilitated the recording of the optical spectra under anaerobic conditions. Subtraction of the absorption spectrum of apo AAP from those of the substituted enzymes was performed using Shimadzu UV-3101 software. Low-temperature dual-mode EPR 
spectroscopy was performed using a Bruker ESP-300E spectrometer equipped with an ER 4116 DM dual-mode Xband cavity and an Oxford Instruments ESR-900 helium-flow cryostat as described previously (15). Background spectra recorded on an EPR tube containing buffer were aligned with and subtracted from experimental spectra as in earlier work (45). All AAP EPR samples contained 20\% 2-propanol by volume. Signals because of oxygen were occasionally observed in both EPR modes. These signals routinely disappeared upon raising the temperature in the helium cryostat to $125 \mathrm{~K}$ for $5 \mathrm{~min}$ and recooling. All spectra were recorded at a modulation frequency of $100 \mathrm{kHz}$ and modulation amplitude of $1.26 \mathrm{mT}(12.6 \mathrm{G})$ with a sweep rate of $10 \mathrm{mT} \mathrm{s}^{-1}$. Parallel- and perpendicular-mode EPR spectra were recorded at microwave frequencies of about 9.37 and $9.65 \mathrm{GHz}$, respectively; precise microwave frequencies were recorded for individual spectra to facilitate $g$ alignment. Other EPR recording parameters are specified in the figure captions for individual samples. EPR simulations were carried out using XSophe (46).

\section{Crystallization.}

AAP was cocrystallized with bestatin using the crystallization conditions reported for the native enzyme (18). Briefly, purified AAP $(10 \mathrm{mg} / \mathrm{mL})$ in $10 \mathrm{mM}$ Tris at pH 8.0, $10 \mathrm{mM} \mathrm{KSCN}, 0.4 \mathrm{M} \mathrm{NaCl}$, and a 4-fold molar excess of bestatin was crystallized by vapor diffusion using $100 \mathrm{mM}$ Tris at $\mathrm{pH} 8.0,100 \mathrm{mM} \mathrm{KSCN}$, and $4.5 \mathrm{M} \mathrm{NaCl}$ as the precipitating solution. Crystals with dimensions $0.7 \times 0.4 \times 0.4 \mathrm{~mm}$ were obtained in 2 days and were shown to be isomorphous with the native crystals. The crystals belong to space group $P 6_{1} 22$ with the following until cell dimensions: $a=b=107.8 \AA, c=102.6 \AA, \alpha=\beta=90^{\circ}, \gamma=120^{\circ}$, and one monomer per asymmetric unit.

\section{Data Collection and Processing.}

Diffraction data were collected at $4{ }^{\circ} \mathrm{C}$ on an R-axis IIC area detector system mounted on a Rigaku RU-200B rotating anode generator operating at $45 \mathrm{kV}$ and $120 \mathrm{~mA}$. One crystal was used for the entire data collection period. The exposures for $25 \mathrm{~min}$ were taken with an oscillation step size of $0.5^{\circ}$. A $0.3-\mathrm{mm}$ collimator was used, and the crystal-detector distance was $100 \mathrm{~mm}$. The diffraction data were integrated and scaled using HKL software (47). Data collection and refinement statistics are outlined in Table 1. The data were collected with an extremely high redundancy; $94.2 \%$ of the reflections were measured 4 times or more. A total of $33 \%$ of the observed reflections were measured 9-12 times. Because the data are so redundant, the $R_{\text {merge }}$ values were raised artificially. With greater redundancy, the correlation between $I$ and $l_{\text {avg }}$ becomes lower and the $R_{\text {merge }}$ increases. The $R_{\text {merge }}$ in the outer shell (2.07-2.0 $\AA$ ) was $68.3 \%$, and the overall $R_{\text {merge }}$ was $14.8 \%$. The $l_{\text {avg }} / \sigma(I)_{\text {avg }}$ was 3.0 in the outer resolution shell.

Table 1: Data Collection and Refinement Statistics

\begin{tabular}{|c|c|}
\hline \multicolumn{2}{|l|}{ Crystal Data } \\
\hline space group & $P 6_{1} 22$ \\
\hline \multirow[t]{3}{*}{ unit cell parameters $(\AA)$} & $a=107.8$ \\
\hline & $b=107.8$ \\
\hline & $c=102.6$ \\
\hline \multicolumn{2}{|l|}{ Data Processing } \\
\hline number reflections, observed & 348645 \\
\hline number reflections, unique & 24377 \\
\hline cuttoff $(I / s)$ & 0 \\
\hline$R_{\text {merge }}{ }^{a}$ (overall) (\%) & 14.8 \\
\hline completeness, overall (\%) & 99.0 \\
\hline highest resolution shell $(\AA)$ & $2.07-2.0$ \\
\hline$R_{\text {merge }}{ }^{a}$ (outer shell) (\%) & 71.4 \\
\hline completeness, outer shell (\%) & 99.8 \\
\hline
\end{tabular}




\begin{tabular}{|c|c|}
\hline \multicolumn{2}{|l|}{ Model Refinement } \\
\hline resolution range $(\AA ̊)$ & $10-2.0$ \\
\hline cuttoff $(F / s F)$ & 0 \\
\hline$R$ factor $^{b}(\%)$ & 19.5 \\
\hline number of reflections & 21037 \\
\hline$R_{\text {free }}$ (for 2053 reflections; \%) & 24.5 \\
\hline number of protein atoms & 2211 \\
\hline number of zinc ions & 2 \\
\hline number of bestatin atoms & 22 \\
\hline number of water molecules & 136 \\
\hline$B$ factor model & individual \\
\hline \multicolumn{2}{|l|}{ RMSD from ideality } \\
\hline bond lengths $(\AA ̊)$ & .006 \\
\hline bond angles (deg) & 1.19 \\
\hline improper angles (deg) & .63 \\
\hline dihedral angles (deg) & 25.6 \\
\hline residues in most favored positions ${ }^{c}(\%)$ & 87.2 \\
\hline
\end{tabular}

${ }^{a} R_{\text {merge }}=\sum\left|l_{\text {obs }}-l_{\text {avg }}\right| / \sum l_{\text {avg. }}{ }^{b} R$ factor $=\sum\left|F_{\text {obs }}-F_{\text {calc }}\right| / \Sigma\left|F_{\text {obs }}\right| .{ }^{c}$ As determined by PROCHECK.

Structure Solution and Refinement. Because crystals of the bestatin-inhibited enzyme were isomorphous with those of the native enzyme, the phases from the published native structure (access code 1AMP) (18) were used as the starting model. In this process, the zinc ions and water molecules were omitted from the original coordinate file. All refinement procedures were carried out using the software package X-PLOR (48). An $R_{\text {free }}(49)$ data set was made prior to any refinement using $9 \%$ of the total reflections. The initial model was subjected to a rigid-body refinement using all reflections in the 20.0-4.0 A resolution range. Subsequent rounds of positional refinement were carried out using higher resolution data incrementally to $2.0 \AA$ resolution. The $R$ factor and $R_{\text {free }}$ at this point were 27.8 and $32.0 \%$, respectively. Difference electron density maps with amplitudes $2 F_{\text {obs }}-F_{\text {calc }}$ and $F_{\text {obs }}-F_{\text {calc }}$ were then calculated and showed clear electron density in the active site for the bound inhibitor and the missing $\mathrm{Zn}$ " ions. The two zinc ions and several water molecules were added to the model, and the model was subjected to further rounds of positional refinement. A model for bestatin was built using Insight (Accelrys, Inc.) and fit to the observed electron density. Additional water molecules were added to the model using the WATERPICK protocol in X-PLOR. Further rounds of positional refinement as well as overall and individual $B$-factor refinement protocols resulted in a final structure with an $R$ factor of $19.5 \%$ and an $R_{\text {free }}$ of 24.5\%. In addition to the 291 amino acid residues, the final model contained 2 zinc ions, 136 water molecules, and 22 atoms of the inhibitor molecule. Simulated annealing omit maps, in which the active site region was omitted, were calculated, which confirmed the presence of the bound inhibitor.

\section{Results}

\section{Electronic Absorption Spectroscopy.}

The electronic absorption spectrum of [CoCo(AAP)] was recorded in both the absence and presence of bestatin (Figure 2), and the absorption because of apo AAP was subtracted in both cases. The spectrum recorded in the absence of the inhibitor is identical to those previously reported for [CoCo(AAP)] (34) and is characterized by a maximum molar absorptivity of $\sim 95 \mathrm{M}^{-1} \mathrm{~cm}^{-1}$ at $545 \mathrm{~nm}$. Upon the addition of 4 equiv of bestatin, the maximum molar absorptivity decreased slightly to $\sim 80 \mathrm{M}^{-1} \mathrm{~cm}^{-1}$, indicating that the Co" coordination number does not change. However, three absorption bands at $495 \mathrm{~nm}\left(83 \mathrm{M}^{-1} \mathrm{~cm}^{-1}\right), 525 \mathrm{~nm}\left(88 \mathrm{M}^{-1} \mathrm{~cm}^{-1}\right)$, and $545 \mathrm{~nm}\left(87 \mathrm{M}^{-}\right.$ ${ }^{1} \mathrm{~cm}^{-1}$ ) appear upon bestatin binding, indicating that bestatin interacts with the dinuclear active site of AAP. 


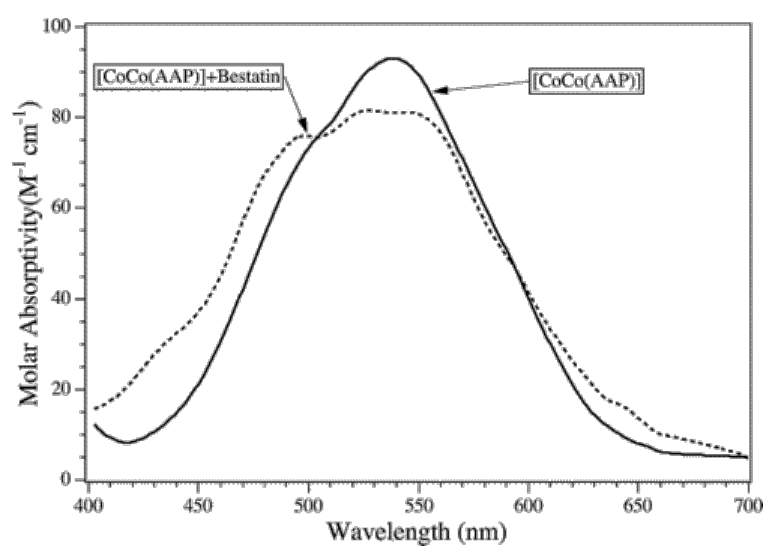

Figure 2 Electronic absorption spectra of a $1 \mathrm{mM}$ sample [CoCo(AAP)] in $50 \mathrm{mM}$ HEPES buffer, at pH 7.5 and 25 ${ }^{\circ} \mathrm{C}$, and $20 \%$ 2-propanol by volume, in the absence $(-)$ and presence $(--)$ of 4 equiv of bestatin.

Electronic absorption spectra of [Co_(AAP)] and [ZnCo(AAP)] were also recorded in the absence and presence of bestatin (Figure 3). Spectra recorded in the absence of bestatin are identical to those previously reported for [Co_(AAP)] and [ZnCo(AAP)] after the subtraction of the absorption because of apo AAP (34). When bestatin was added to [Co_(AAP)], the maximum molar absorptivity increased from $\sim 50$ to $58 \mathrm{M}^{-1} \mathrm{~cm}^{-1}$ and the broad absorption band observed for [Co_(AAP)] was replaced by three, resolved absorption bands at $495 \mathrm{~nm}^{(} 54 \mathrm{M}^{-}$ $\left.{ }^{1} \mathrm{~cm}^{-1}\right), 520 \mathrm{~nm}\left(58 \mathrm{M}^{-1} \mathrm{~cm}^{-1}\right)$, and $545 \mathrm{~nm}\left(45 \mathrm{M}^{-1} \mathrm{~cm}^{-1}\right)$, similar to the changes observed for [CoCo(AAP)]. On the other hand, when bestatin is added to [ZnCo(AAP)], the overall shape of the absorption band did not change but the maximum molar absorptivity increased slightly from $\sim 30$ to $35 \mathrm{M}^{-1} \mathrm{~cm}^{-1}$ and the absorption band is blueshifted from 530 to $520 \mathrm{~nm}$.

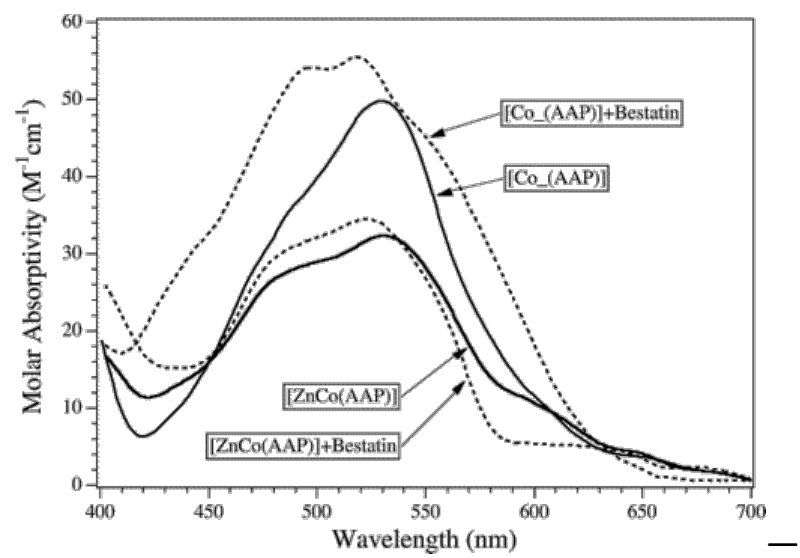

Figure 3 Electronic absorption spectra of $1 \mathrm{mM}$ samples of [Co_(AAP)] and [ZnCo(AAP)] in $50 \mathrm{mM}$ HEPES buffer, at pH 7.5 and $25^{\circ} \mathrm{C}$, and $20 \%$ 2-propanol by volume, in the absence (-) and presence (- - -) of 4 equiv of bestatin.

\section{EPR Spectroscopy.}

The EPR spectrum of [CoCo(AAP)] is shown in Figure 4. Upon the addition of 4 equiv of bestatin, the perpendicular-mode signal observed at $10 \mathrm{~K}$ resulting from the two $S=3 / 2 \mathrm{Co}$ " ions is quenched. Examination of the parallel-mode EPR spectrum of [CoCo(AAP)]-bestatin revealed no detectable parallel-mode signal. The EPR spectrum of [CoZn(AAP)] and [ZnCo(AAP)] was also recorded with and without added bestatin (Figure 5). Noticeable differences are present in the spectra of both heterodimetallic forms of AAP upon binding bestatin, namely, the appearance of distinct hyperfine splitting in both samples. The spectra of [CoZn(AAP)] and [ZnCo(AAP)] were also examined at higher temperatures and powers. The maximum normalized signal intensity 
was observed at low temperatures $(\sim 5 \mathrm{~K})$ and low microwave powers, indicating that the observed signals are likely the result of an $M_{s}=| \pm 1 / 2\rangle$ ground-state transition. The observed signals are distorted by saturation at higher powers ( $\geq 20 \mathrm{~mW}$ ) and by relaxation at higher temperatures $(\geq 20 \mathrm{~K})$, and consequently, multiple species could not be reliably resolved by subtraction of spectra recorded under different conditions. However, computer simulation of the EPR signal exhibited by [CoZn(AAP)]-bestatin (Figure 5) suggests that two species are present. One species is axial with no resolved hyperfine structure. The EPR spectrum observed for this species was simulated assuming an isotropic $g$ factor and axial zero-field splitting $\left(H=S D S+6 B g S ; S=3 / 2, g_{\text {iso }}=\right.$ $2.25, D \gg h v, E / D=0)$. The second species was highly rhombic and exhibited clearly resolved ${ }^{59} \mathrm{Co}$ hyperfine splitting $\left(H=S D S+B B g S+S A I ; S={ }^{3} / 2, I\left({ }^{59} \mathrm{Co}\right)=7 / 2, g_{\text {iso }}=2.38, D \gg h v, E / D=0.24, A\left({ }^{59} \mathrm{Co}\right)=8.1 \times 10^{-3} \mathrm{~cm}^{-1}\right)$. On the other hand, the EPR spectra of [ZnCo(AAP)] in the absence and presence of bestatin consisted of multiple unresolved features. Even so, some clear features are present in the EPR spectrum of the [ZnCo(AAP)]-bestatin complex that are absent in the EPR spectrum of [ZnCo(AAP)]. In particular, a high-field shoulder centered at $g_{\text {eff }} \sim 1.78(3840 \mathrm{G})$ and a clear hyperfine pattern centered at $g_{\text {eff }} \sim 7.8(945 \mathrm{G})$ suggest the formation of a distinct species, with very high rhombicity $(E / D \sim 1 / 3)$, low $g$ strain, and modest spin-orbit coupling $\left(g_{\text {real }} \sim 2.4\right)$, upon the addition of bestatin.

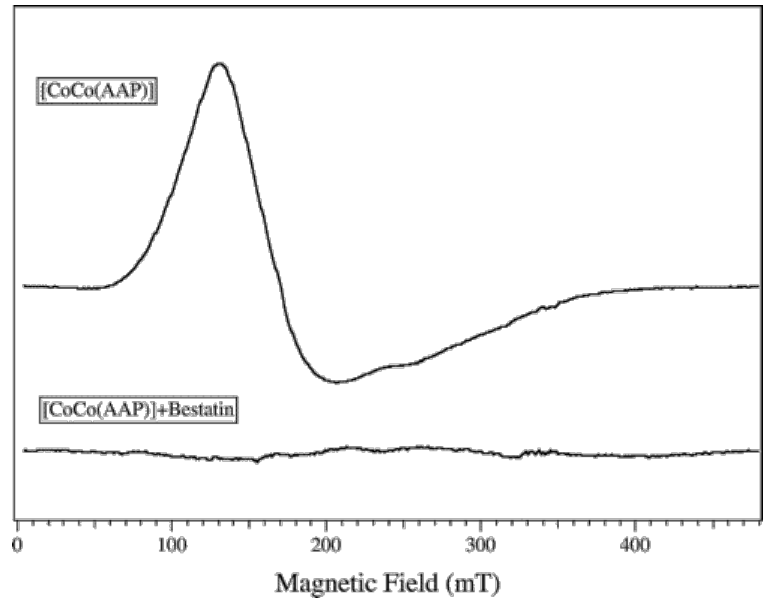

Figure 4 Perpendicular-mode EPR signal of [CoCo(AAP)] in $50 \mathrm{mM}$ HEPES buffer at pH 7.5 in the presence (-) and absence (- - -) of 4 equiv of bestatin. All spectra were recorded at $10 \mathrm{~K}, 0.2 \mathrm{~mW}, 1.26 \mathrm{mT}$ modulation amplitude, and $10 \mathrm{mT} \mathrm{s}^{-1}$ sweep rate with $20 \%$ added 2-propanol by volume.
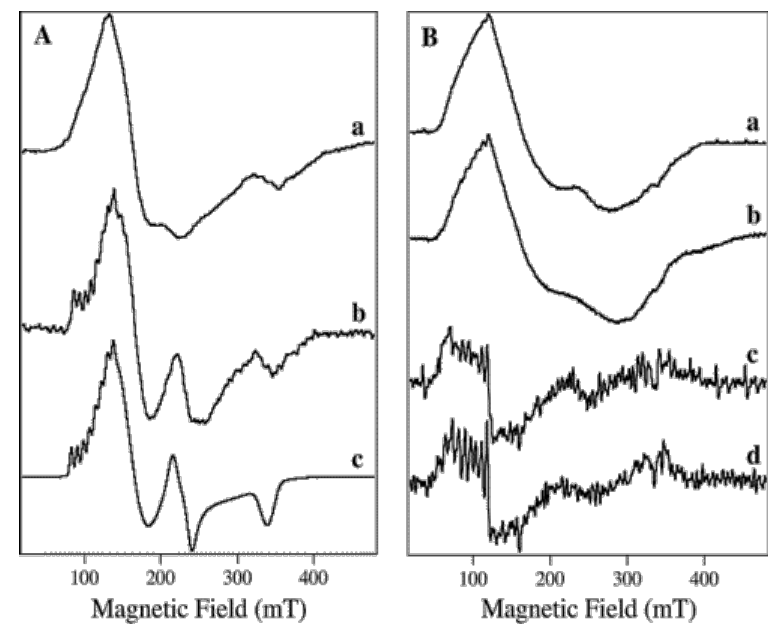

Figure 5 (A) Perpendicular-mode EPR spectra of (a) [CoZn(AAP)], (b) [CoZn(AAP)] + bestatin, and (c) computer simulation of b. (B) Perpendicular-mode EPR spectra of (a) [ZnCo(AAP)], (b) [ZnCo(AAP)] + bestatin, (c) 
[ZnCo(AAP)], and (d) [ZnCo(AAP)] + bestatin. All spectra were recorded at $10 \mathrm{~K}, 0.2 \mathrm{~mW}, 1.26 \mathrm{mT}$ modulation amplitude, and $10 \mathrm{mT} \mathrm{s}^{-1}$ sweep rate with $20 \%$ added 2-propanol by volume.

\section{X-ray Structure of Bestatin-Bound AAP.}

The X-ray crystal structure of the [ZnZn(AAP)]-bestatin complex was solved to $2.0 \AA$ resolution and is shown in Figure 6. Inhibitor binding does not introduce major conformational changes to the overall protein structure. The structures of $[\mathrm{ZnZn}(\mathrm{AAP})]$ and the [ZnZn(AAP)]-bestatin complex agree with an rms deviation of $0.19 \AA$ for the 291 structurally equivalent $C \alpha$ atoms. Both side chains of bestatin occupy the well-defined hydrophobic pocket adjacent to the dinuclear Zn" active site made up of Leu155, Met180, Ile193, Cys223, Tyr225, Cys227, Met242, Phe244, Phe248, Tyr251, and Ile255. The leucine portion of bestatin extends back toward Leu155, while the phenylalanine ring extends toward Phe244, Tyr251, and Phe248. The C terminus of bestatin forms a hydrogen bond with Tyr225 (2.7 ̊), while the two Cys residues at the back of the hydrophobic pocket (Cys223 and Cys227) form a disulfide bond.
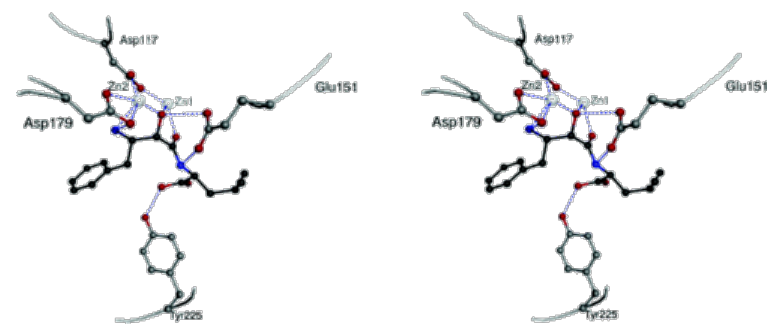

Figure 6 Stereo figure of the active site region of [ZnZn(AAP)] with bestatin bound. The His residues that act as $\mathrm{Zn}$ "ligands have been omitted for clarity.

The amino acid residues ligated to the dizinc(II) cluster in AAP are identical to those in the native structure with only minor perturbations to the bond lengths (Table 2; Figure 7). Zn1 of the [ZnZn(AAP)]-bestatin complex exhibits a distorted trigonal bipyramidal geometry with the $N^{\varepsilon}$ nitrogen of His 256 and the alkoxide oxygen atom of bestatin making up the axial positions. On the other hand, $\mathrm{Zn} 2$ resides in a distorted trigonal bipyramidal geometry with the $\mathrm{N}^{\varepsilon}$ nitrogen of His 97 and the $\mathrm{N}$-terminal amine nitrogen atom of bestatin making up the axial positions. The angle including $\mathrm{N}_{\text {amine }}-\mathrm{Zn2}-\mathrm{N}^{\varepsilon}$ is $161^{\circ}$. Glu152 is coordinated in an asymmetric bidentate fashion to $\mathrm{Zn} 1$ with the additional oxygen atom providing a potential sixth ligand at a distance of $2.3 \AA$. Similarly, Asp179 is coordinated to $\mathrm{Zn} 2$ in an asymmetric bidentate fashion with the second oxygen atom residing $2.4 \AA$ from the metal center, providing a potential sixth ligand. The alkoxide oxygen of bestatin bridges between the two $\mathrm{Zn}^{\prime \prime}$ ions in the active site displacing the bridging water molecule observed in the native [ZnZn(AAP)] structure. The M-M distance for the AAP-bestatin complex is identical to that in native AAP (3.5 $\AA$ ) with alkoxide oxygen atom distances of 2.1 and $1.9 \AA$ from Zn1 and Zn2, respectively. Interestingly, the backbone carbonyl oxygen atom of bestatin is coordinated to $\mathrm{Znl}$ at a distance of $2.3 \AA$. In addition, the $\mathrm{NH}_{2}$ group of bestatin, which mimics the $\mathrm{N}$-terminal amine group of an incoming peptide, binds to $\mathrm{Zn} 2$ with a bond distance of $2.3 \AA$. Two hydrogen bonds exist between Glu151 and the bestatin molecule. One of the Glu151 oxygen atoms is $3.0 \AA$ from the alkoxide moiety of bestatin, while the second is $3.3 \AA$ from the backbone amide of the inhibitor. Another important hydrogen-bonding interaction is also observed between an oxygen atom of Asp99 and the $\mathrm{N}^{\delta}$ nitrogen of His97 (3.1 $\AA$ ) forming an Asp-His-Zn triad (50). This distance is longer than those observed in [ZnZn(AAP)] (2.8 $\AA ̊$ ), [ZnZn(AAP)]-1-butaneboronic acid (BuBA) (2.7 Å), and [ZnZn(AAP)]-I-leucine phosphonic acid (LPA) (2.9 ̊̊). 


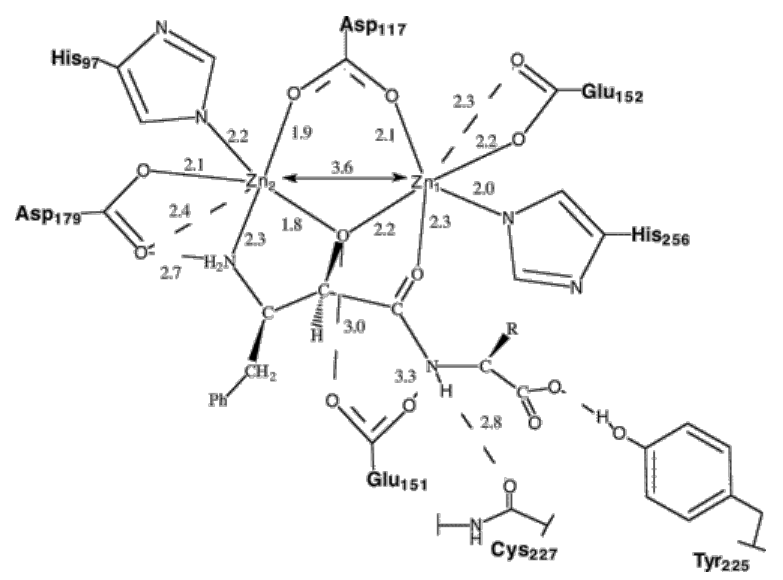

Figure 7 Schematic of bestatin bound to [ZnZn(AAP)] showing all distances in angstroms.

Table 2: Selected Bond Lengths (Angstroms) for the Zinc-Ligand Distances in the AAP-Bestatin Complex

\begin{tabular}{|l|l|l|l|l|}
\hline zinc-ligand distance $(\AA)$ & & & & \\
\hline & native & BuBA complex & LPA complex & bestatin complex \\
\hline Zn1-Zn2 & 3.5 & 3.3 & 3.9 & 3.5 \\
\hline Zn1-Asp117 O2 & 2.1 & 2.2 & 1.9 & 2.0 \\
\hline Glu152 01 & 2.0 & 2.2 & 2.0 & 2.3 \\
\hline Glu152 O2 & 2.4 & 2.6 & 2.4 & 2.2 \\
\hline His256 N2 & 2.3 & 2.3 & 2.1 & 2.1 \\
\hline $\mathrm{H}_{2} \mathrm{O}$ & 2.3 & & & \\
\hline BuBA O1 & & 2.5 & & \\
\hline BuBA O2 & & 2.7 & & \\
\hline LPA O1 & & & 1.9 & \\
\hline bestatin O1 & & & & 2.1 \\
\hline bestatin O2 & & & & 2.3 \\
\hline Zn2-Asp117 O1 & 2.0 & 2.1 & 2.0 & 2.0 \\
\hline Asp179 O1 & 2.1 & 2.2 & 2.2 & 2.2 \\
\hline Asp179 O2 & 2.3 & 2.5 & 2.4 & 2.4 \\
\hline His97 N2 & 2.3 & 2.2 & 2.2 & 2.2 \\
\hline $\mathrm{H}_{2} \mathrm{O}$ & 2.3 & & & \\
\hline BuBA O2 & & 3.0 & & \\
\hline BuBA O1 & & 4.4 & & \\
\hline LPA O3 & & & 2.3 & \\
\hline LPA NH 2 & & & & \\
\hline bestatin O1 & & & & 2.1 \\
\hline bestatin NH $H_{2}$ & & & \\
\hline
\end{tabular}

\section{Discussion}

Electronic absorption spectra of the catalytically competent [Co_(AAP)], [CoCo(AAP)], and [ZnCo(AAP)] enzymes recorded in the absence and presence of bestatin suggest that, like the bILAP-bestatin complex (35), both of the divalent metal ions in AAP are involved in binding bestatin. The molar absorptivities observed for each active site Co" ion are also consistent with five-coordinate metal ions; however, the first metal-binding site is much more perturbed than the second. Interestingly, the electronic perturbations observed for [Co_(AAP)] and [ZnCo(AAP)], 
upon the addition of bestatin, are essentially opposite to those observed for LPA binding to Co"-substituted AAP $(51,52)$. The X-ray crystal structure of the [ZnZn(AAP)]-LPA complex revealed that LPA interacts with both metal centers, but a single oxygen-atom bridge is absent (Figure 1C) (52). However, the N-terminal amine of LPA binds to the second metal center with a bond distance of $2.1 \AA$ A. Thus, LPA binds to AAP as an $\eta-1,2-\mu$-phosphonate with one and two phosphonate oxygen atom ligand to the first and second metal ions, respectively, along with the $\mathrm{N}$-terminal amine nitrogen atom bound to $\mathrm{Zn2}$. On the basis of these data, the electronic absorption spectra of Co"-substituted forms of AAP in the presence of bestatin are consistent with bestatin contributing two strongly bound ligands to the first metal ion but only one strong ligand to the second metal-binding site.

EPR spectra of [CoCo(AAP)] in the absence and presence of bestatin were recorded in both the perpendicular and parallel modes. In the absence of bestatin, the perpendicular-mode EPR spectrum reveals an $M_{s}=| \pm 1 / 2\rangle$ ground-state transition with an isotropic $g_{\text {real }}=2.25$ and $E / D=0.095$, which accounts for only $13 \%$ of the expected spin density, suggesting that the two Co" ions are spin-coupled in the majority of enzyme molecules, as previously reported (43). A parallel-mode signal is observed at neutral $\mathrm{pH}$ but is abolished at $\mathrm{pH} 10$. These data were interpreted as being a consequence of there being a weakly bound $\mu-\left(\mathrm{H}_{2} \mathrm{O}\right)$ bridge between the metal ions in the active site of the resting enzyme and a strongly bound $\mu-(\mathrm{OH})$ bridge in the inactive enzyme at high $\mathrm{pH}$ (20). The addition of bestatin to [CoCo(AAP)] completely abolished the perpendicular-mode signal. These data suggest that the two $\mathrm{Co}^{\prime \prime}$ ions in the AAP-bestatin complex are strongly spin-coupling; therefore, bestatin binds to both $\mathrm{Co}^{\prime \prime}$ ions. Examination of the [CoCo(AAP)]-bestatin complex in the parallel mode provided no detectable signal over a broad range of temperatures and powers. These data suggest that the two Co" ions in AAP are antiferromagnetically coupled yielding an $S=0$ ground state. On the basis of the reported magnetic properties of several $\mu$-aquo or $\mu$-hydroxodicobalt(II) model complexes, one would expect weak to moderately strong antiferromagnetic coupling in systems containing a single oxygen-atom bridge (53-55). Conversely, systems lacking a strongly bound single atom bridge, such as bis( $\mu$-carboxylato) and tetrakis( $\mu$-carboxylato)dicobalt(II) cores, do not exhibit strong antiferromagnetic spin coupling (56-58). Interestingly, the ( $\mu$ phosphonato)dicobalt(II) core of the AAP-LPA complex exhibits ferromagnetic coupling $(48,49)$. A combination of these data suggests that a single oxygen-atom bridge is present in the bestatin-AAP complex that can be assigned to bestatin alkoxide group, similar to the bILAP-bestatin structure (35).

EPR data obtained on heterodimetallic forms of AAP ([CoZn(AAP)] and [ZnCo(AAP)]) serve both to confirm that bestatin interacts with both metal ions and to support the contention that a bestatin-derived single oxygenatom bridge exists between the Co"l ions in solution as well as in the crystalline state. EPR spectra of [CoZn(AAP)] and [ZnCo(AAP)] consist of a pH-dependent mixture of two species (44). For each of these heterodimetallic forms of AAP, an axial species with low spin-orbit coupling and high $g$ strain dominates at $\mathrm{pH} 7-8$, suggesting that the active nucleophile is present as a water molecule. At higher $\mathrm{pH}$, a rhombic species with a high degree of spin-orbit coupling and very little $g$ strain, indicated by the well-resolved ${ }^{59} \mathrm{Co}$ hyperfine structure, is the dominant species. This species is, therefore, electronically asymmetric and highly geometrically constrained as would be expected for a strongly bound bridging ligand such as $\mu-\mathrm{OH}$. Upon the addition of bestatin to [CoZn(AAP)] and [ZnCo(AAP)], there is clearly an increase in the proportions of the rhombic species exhibiting the well-resolved hyperfine structure relative to the poorly resolved axial species. These data indicate that bestatin interacts with both metal ions and strongly suggest the replacement of water with a more strongly bound ligand such as the alkoxide functionality of bestatin.

Spectroscopic studies suggest that bestatin bridges between the two active site metal ions of AAP in solution via a $\mu$-alkoxide moiety but provides no detailed information regarding any interaction of the $\mathrm{N}$-terminal amine with the second divalent metal ion in the active site. To further characterize the binding of bestatin to AAP, the enzyme was crystallized in the presence of bestatin and the X-ray crystal structure was determined to $2.0 \AA$ resolution (Figures 6 and 7). The AAP-bestatin complex provides structural information that can be used as a 
model for inhibitor design, as well as the enzyme-substrate complex. Like all of the structures reported for AAP-inhibitor complexes, the phenyl group of bestatin resides in a well-defined hydrophobic pocket adjacent to the dinuclear $\mathrm{Zn}$ " active sites $(18,59)$. This hydrophobic pocket has been shown to be the initial substrate recognition and binding point on the enzyme, which constitutes the first step in the catalytic mechanism (60). Bestatin binding to AAP does not alter the $\mathrm{Zn}$ " $-\mathrm{Zn}$ " distance but does change the coordination number of both $\mathrm{Zn}$ " ions to five coordinate, consistent with the electronic absorption data.

No active site ligands are displaced upon bestatin binding except the water molecule that bridges the two $\mathrm{Zn}$ " ions in the wild-type structure. This bridging water molecule is displaced by the alkoxide oxygen of bestatin, consistent with EPR data obtained on the [CoCo(AAP)]-bestatin complex. Interestingly, the Zn1-O1 distance is $2.1 \AA$, while the $\mathrm{Zn} 2-\mathrm{O}$ distance is $1.9 \AA$. Therefore, the alkoxide oxygen atom appears to interact more strongly with $\mathrm{Zn} 2$ than with $\mathrm{Zn} 1$, suggesting that $\mathrm{Zn} 2$ plays a role in substrate activation as well as stabilization of the transition state. The bestatin carbonyl group (O2) binds to Zn1, albeit weakly, with a bond distance of $2.3 \AA$. Surprisingly, the N-terminal amine nitrogen of bestatin (N1) resides $2.3 \AA$ from Zn2, suggesting that it is not coordinated or is very weakly interacting with $\mathrm{Zn2}$. These data are consistent with both the electronic absorption and EPR data, indicating that the two metal ions in the AAP-bestatin complex are perturbed in a very similar manner but that the proportion of the "new" species is much higher for M1 than for M2. The EPR data also show that $\mathrm{M} 2$ of the AAP-bestatin complex is not as perturbed as M2 in the AAP-LPA complex. A comparison to the transition-state analogue inhibitor structure of [ZnZn(AAP)]-LPA suggests that the bestatin structure best represents the substrate-binding step and further supports the proposal that carbonyl binding occurs before $\mathrm{N}$-terminal amine binding to the dinuclear active site $(61,62)$.

The orientation of the N-terminal amine group in the AAP-bestatin structure is similar to the AAP-LPA structure, but the electron orbital overlap observed between $\mathrm{Zn} 1$ and the $\mathrm{N}$-terminal amine nitrogen atom in the AAP-bestatin complex is smaller, resulting in a longer M2-amine-N1 distance. However, the orientation of the $\mathrm{N}$-terminal amine of bestatin is distinctly different than that observed for AAP bound by $\mathrm{d}$ iodophenylalaninehydroxamate (d-IPH) (59). In the d-IPH complex, the N-terminal amine group is not present in the active site pocket but instead forms a hydrogen bond with Tyr-225 near the surface of the enzyme at the mouth of the active site pocket. Conversely, the N-terminal amine in the LPA complex is coordinated to Zn2 with a bond distance of $2.1 \AA$, while it resides $2.4 \AA$ away from Zn2 in the bestatin structure. Therefore, the inhibitor conformation observed in the AAP-d-IPH complex is an inaccurate representation of the actual transition state of peptide hydrolysis, i.e., the stereochemistry of the d-IPH inhibitor is incorrect for an actual peptide because $\mathrm{IPH}$ resides in the $\mathrm{d}$ configuration. With this in mind, little mechanistic information can be gleaned from the [ZnZn(AAP)]-d-IPH structure because $d$-IPH is simply acting as a metal chelator, rather than mimicking either substrate binding or the transition state of the catalytic reaction. Consequently, any mechanistic conclusions derived from the AAP-d-IPH structure likely led to an incorrect assignment for the role of the second metal ion in the peptide hydrolysis reaction catalyzed by $\operatorname{AAP}(37,43)$. On the basis of the structures of the AAP-bestatin and AAP-LPA complexes, Tyr225 is not involved in the binding the N-terminal amine of the peptide substrate. In fact, this residue is not likely involved mechanistically but, instead, may contribute only as another large hydrophobic residue used to line the active site pocket.

Glu151 of the active site pocket has been implicated in the catalytic mechanism based on the close proximity of its side chain to the bridging water molecule in the native enzyme (59) and to the bridging phosphonate moiety of LPA in the AAP-LPA complex (52). In the AAP-bestatin complex, there are two hydrogen-bonding interactions between Glu151 and the inhibitor. One Glu151 oxygen atom is $3.0 \AA$ from the $\mu$-alkoxide moiety of bestatin, while the second is $3.3 \AA$ from the backbone amide of the inhibitor. Glu151 appears to be well-positioned to function as a proton shuttle in the hydrolytic reaction, where it could be responsible for removing and/or delivering the proton that is transferred to the leaving group (63). It should be noted, however, that bestatin 
contains an additional hydroxyl group between the backbone amine and the carbonyl carbon as compared to the natural substrate. Therefore, interactions with Glu151 may differ for substrates versus bestatin, but the interactions observed in the AAP-bestatin complex suggest that two hydrogen-bonding interactions are possible with Glu151. Furthermore, the AAP-bestatin structure suggests that Glu151 can transfer a proton from the nucleophilic hydroxide moiety to the new $\mathrm{N}$-terminal amine. Such a proton transfer must occur because the newly formed $\mathrm{N}$-terminal amine must leave, at a minimum, as an $\mathrm{NH}_{2}$ group.

In conclusion, we have spectroscopically and crystallographically characterized the binding of bestatin to AAP. Because bestatin has been shown to be an effective drug to inhibit matrix degradation and invasion of extracellular matrixes by fibrosarcoma cells as well as decrease HIV viral load in males $(6,7)$, the binding mode of bestatin to AAP provides a structural foundation for inhibitor design. In addition, mechanistic data pertaining to the substrate-binding step in peptide hydrolysis can also be gleaned. The data presented herein are consistent with backbone carbonyl binding to $\mathrm{Zn} 1$ followed by $\mathrm{N}$-terminal amine binding to $\mathrm{Zn} 2$. Because AAP is $80 \%$ active with only one divalent metal ion, carbonyl binding likely occurs at $\mathrm{Zn} 1$. However, the short Zn2-01 bond distance of $1.8 \AA$ suggests that, if a second divalent metal ion is present, it likely assists in the activation of the backbone carbonyl, making the carbonyl carbon susceptible to nucleophilic attack. Furthermore, these data suggest that Glu151 is the active site acid/base and can transfer a proton to the newly formed $\mathrm{N}$-terminal amine.

\section{References}

1 Taylor, A. (1993) Aminopeptidases: Structure and Function, FASEB J.7, 290.

2 Taylor, A. (1996) Aminopeptidases, in Molecular Biology Intelligence Unit (Taylor, A., Ed.) pp 1, R. G. Landes Co., Austin, TX.

3 Gonzales, T., and Bobert-Baudouy, J. (1996) Bacterial Aminopeptidases: Properties and Functions, FEMS Microbiol. Rev.18, 319.

4 Passani, L. A., Vonsattel, J. P., Carter, R. E., and Coyle, J. T. (1997) N-Acetylaspartylglutamate, NAcetylaspartate, and $N$-Acetylated $\alpha$-Linked Acidic Dipeptidase in Human Brain and Their Alterations in Huntington and Alzheimer's Diseases, Mol. Chem. Neuropathol.31, 97.

5 Aoyagi, T., Suda, T., Nagai, M., Ogawa, K., Suzuki, J., Takeuchi, T., and Umezawa, H. (1976) Aminopeptidase Activities on the Surfase of Mammalian Cells, Biochim. Biophys. Acta452, 131.

6 Pulido-Cejudo, G., Conway, B., Proulx, P., Brown, R., and Izaguirre, C. A. (1997) Bestatin-Mediated Inhibition of Leucine Aminopeptidase May Hinder Hiv Infection, Antiviral Res.36, 167.

7 Fujii, H., Nakajima, M., Aoyagi, T., and Tsuruo, T. (1996) Inhibition of Tumor Cell Invasion and Matrix Degradation by Aminopeptidase Inhibitors, Biol. Pharm. Bull.19, 6.

$8 \mathrm{Holz}$, R. C., Bzymek, K., and Swierczek, S. I. (2003) Co-Catalytic Metallopeptidases as Pharmaceutical Targets, Cur. Opin. Chem. Biol.7, 197.

9 Bradshaw, R., and Yi, E. (2002) Methionine Aminopeptidases and Angiogenesis, Essays Biol. Med.38, 65.

10 Daiyasua, H., Osakaa, K., Ishinob, Y., and Toha, H. (2001) Expansion of the Zinc Metallo-Hydrolase Family of the I-Lactamase Fold, FEBS Lett.503, 1.

11 Lipscomb, W. N., and Sträter, N. (1996) Recent Advances in Zinc Enzymology, Chem. Rev.96, 2375.

12 Wilcox, D. E. (1996) Binuclear Metallohydrolases, Chem. Rev.96, 2435.

13 Dismukes, G. C. (1996) Manganese Enzymes with Binuclear Active Sites, Chem. Rev.96, 2909.

$14 \mathrm{Holz}, \mathrm{R}$. C. (2002) The Aminopeptidase from Aeromonas proteolytica: Structure and Mechanism of CoCatalytic Metal Centers Involved in Peptide Hydrolysis, Coord. Chem. Rev.232, 5.

15 Barrett, A. J., Rawlings, N. D., and Woessner, J. F. (1998) Handbook of Proteolytic Enzymes, pp 1, Academic Press, London, U.K.

16 Velasco, A. M., Leguina, J. I., and Lazcano, A. (2002) Molecular Evolution of the Lysine Biosynthetic Pathways, J. Mol. Evol.55, 445. 
17 Remaut, H., Bompard-Gilles, C., Goffin, C., Frere, J.-M., and Van Beeumen, J. (2001) Structure of the Bacillussubtilisd-Aminopeptidase Dppa Reveals a Novel Selfcompartmentalizing Protease, Nat. Struct. Biol.8, 674.

18 Chevrier, B., Schalk, C., D'Orchymont, H., Rondeau, J.-M., Moras, D., and Tarnus, C. (1994) Crystal Structure of Aeromonasproteolytica Aminopeptidase: A Prototypical Member of the Co-Catalytic Zinc Enzyme Family, Structure2, 283.

19 Desmarais, W., Bienvenue, L. D., Bzymek, K., Holz, R. C., Petsko, A. G., and Ringe, D. (2002) The $0.95 \AA$ Resolution and Low pH Crystal Structures of the Aminopeptidase from Aeromonasproteolytica, Biochemistry, in press.

20 Desmarais, W., Bienvenue, L. D., Bzymek, K., Holz, R. C., Petsko, A. G., and Ringe, D. (2002) The $1.2 \AA$ Resolution Crystal Structure of the Aminopeptidase from Aeromonasproteolytica Complexed with Tris: A Tale of Buffer Inhibition, Structure 10, 1063.

21 Baker, J. O., and Prescott, J. M. (1983) Aeromonas Aminopeptidase: pH Dependence and a Transition-StateAnalogue Inhibitor, Biochemistry22, 5322.

22 Baker, J. O., Wilkes, S. H., Bayliss, M. E., and Prescott, J. M. (1983) Hydroxamates and Aliphatic Boronic Acids: Marker Inhibitors for Aminopeptidase, Biochemistry22, 2098.

23 Baker, J. O., and Prescott, J. M. (1985) A Transition-State-Analogue Inhibitor Infuences Zinc-Binding by Aeromonas Aminopeptidase, Biochem. Biophys. Res. Commun.130, 1154.

24 Prescott, J. M., and Wilkes, S. H. (1976) Aeromonas Aminopeptidase, Methods Enzymol.45B, 530.

25 Lejczak, B., Kafarski, P., and Zygmunt, J. (1989) Inhibition of Aminopeptidases by Aminophosphonates, Biochemistry28, 3549.

26 Bennett, B., and Holz, R. C. (1998) Inhibition of the Aminopeptidase from Aeromonasproteolytica by ILeucinephosphonic Acid, a Transition State Analouge of Peptide Hydrolysis, J. Am. Chem. Soc.120, 12139.

27 Sträter, N., and Lipscomb, W. N. (1995) Transition State Analogue I-Leucinephosphonic Acid Bound to Bovine Lens Leucine Aminopeptidase: X-ray Structure at $1.65 \AA$ Resolution in a New Crystal Form, Biochemisty34, 9200.

28 Wilkes, S. H., and Prescott, J. M. (1983) Stereospecifity of Amino Acid Hydroxamate Inhibition of Aminopeptidases, J. Biol. Chem.258, 13517.

29 Wilkes, S. H., and Prescott, J. M. (1987) Hydroxamate-Induced Spectral Perturbations of Cobalt Aeromonas Aminopeptidase, J. Biol. Chem.262, 8621.

30 Chan, W. W.-C., Dennis, P., Demmer, W., and Brand, K. (1982) Inhibition of Leucine Aminopeptidase by Amino Acid Hydroxamates, J. Biol. Chem.257, 7955.

31 Umezawa, H. T., Aoyagi, T., Suda, H., Hamada, M., and Takeuchi, T. (1976) Bestatin, An Inhibitor of Aminopeptidase B, Produced by Actinomycetes, J. Antibiot. 29, 97.

32 Taylor, A., Peltier, C. Z., Torre, F. J., and Hakamian, N. (1993) Inhibition of Bovine Lens Leucine Aminopeptidase by Bestatin: Number of Binding Sites and Slow Binding of This Inhibitor, Biochemistry32, 784.

33 Huntington, K. M., Bienvenue, D., Wei, Y., Bennett, B., Holz, R. C., and Pei, D. (1999) Slow-Binding Inhibition of the Aminopeptidase from Aeromonas proteolytica by Peptide Thiols: Synthesis and Spectral Characterization, Biochemistry38, 15587.

34 Wilkes, S. H., and Prescott, J. M. (1985) The Slow, Tight Binding of Bestatin and Amastatin to Aminopeptidases, J. Biol. Chem.260, 13154.

35 Burley, S. K., David, P. R., Sweet, R. M., Taylor, A., and Lipscomb, W. N. (1992) Structure Determination and Refinement of Bovine Lens Leucine Aminopeptidase and Its Complex with Bestatin, J. Mol. Biol.224, 113.

36 Prescott, J. M., and Wilkes, S. H. (1976) Aeromonas Aminopeptidase, Methods Enzymol.45, 530.

37 Chen, G., Edwards, T., D'souza, V. M., and Holz, R. C. (1997) Mechanistic Studies on the Aminopeptidase from Aeromonasproteolytica: A Two-Metal Ion Mechanism for Peptide Hydrolysis, Biochemistry36, 4278. 
38 Prescott, J. M., Wilkes, S. H., Wagner, F. W., and Wilson, K. J. (1971) Aeromonas Aminopeptidase Improved Isolation and Some Physical Properties, J. Biol. Chem.246, 1756.

39 Edelhoch, H. (1967) Spectroscopic Determination of Tryptophan and Tyrosine in Proteins, Biochemistry6, 1948-1954.

40 Gill, S. C., and von Hippel, P. H. (1989) Calculation of Protein Extinction Coefficients from Amino Acid Sequence Data, Anal. Biochem.182, 319-366.

41 Gill, S. C., Yager, T. D., and von Hippel, P. H. (1990) Thermodynamic Analysis of the Transcription Cycle in $E$. coli, Biophys. Chem.37, 239-250.

42 Prescott, J. M., Wagner, F. W., Holmquist, B., and Vallee, B. L. (1985) Spectral and Kinetic Studies of MetalSubstituted Aeromonas Aminopeptidase: Nonidentical, Interacting Metal-Binding Sites, Biochemistry24, 5350.

43 Bennett, B., and Holz, R. C. (1997) Epr Studies on the Mono- and Dicobalt(II)-Substituted Forms of the Aminopeptidase from Aeromonas proteolytica. Insight into the Catalytic Mechanism of Dinuclear Hydrolases, J. Am. Chem. Soc.119, 1923.

44 Bennett, B., and Holz, R. C. (1997) Spectroscopically Distinct Cobalt(II) Sites in Heterodimetallic Forms of the Aminopeptidase from Aeromonas proteolytica: Characterization of Substrate Binding, Biochemistry36, 9837.

45 Bennett, B., Berks, B. C., Ferguson, S. J., Thomson, A. J., and Richardson, D. J. (1994) Mo(V) Electron Paramagnetic Resonance Signals from the Periplasmic Nitrate Reductase of Thiosphaera pantotropha, Eur. J. Biochem.226, 789.

46 Wang, D. M., and Hanson, G. R. (1995) XSophie, Bruker Biospin, J. Magn. Reson., Ser. A117, 1.

47 Otwinowski, Z., and Minor, W. (1997) Hkl X-Ray Methods, Methods Enzymol.276, 307.

48 Brünger, A. T. (1997) X-PLOR, a System for X-Ray Crystallography and NMR, Yale University Press, New Haven, CT.

49 Brunger, A. T. (1992) Free $R$ Value: A Novel Statistical Quantity for Assessing the Accuracy of Crystal Structures, Nature355, 472-475.

50 Christianson, D. W., and Alexander, R. S. (1989) Carboxylate-Histidine-Zinc Interactions in Protein Structure and Function, J. Am. Chem. Soc.111, 6412.

51 Bennett, B., and Holz, R. C. (1998) Inhibition of the Aminopeptidase from Aeromonasproteolytica by ILeucinephosphonic Acid, a Transition State Analogue of Peptide Hydrolysis, J. Am. Chem. Soc.120, 12139.

52 Stamper, C., Bennett, B., Edwards, T., Holz, R. C., Ringe, D., and Petsko, G. (2001) Inhibition of the Aminopeptidase from Aeromonas proteolytica by I-Leucinephosphonic Acid. Spectroscopic and Crystallographic Characterization of the Transition State of Peptide Hydrolysis, Biochemistry40, in press.

53 Turpeinen, U., Ahlgren, M., and Hämäläinen, R. (1982) Structures of Di- $m$-acetato- $\left(O, O^{\prime}\right)$ - $m$-aquabis[acetato $\left(N, N, N^{\prime}, N^{\prime}\right.$-tetramethylethylenediamine)cobalt(II) and $m$-Aqua-di- $m$-chloroacetato- $\left(O, O^{\prime}\right)$ bis[chloroacetato( $N, N, N^{\prime}, N^{\prime}$-tetramethylethylenediamine)cobalt(II), Acta Crystallogr., Sect.B 32, 1580.

54 Turpeinen, U., Hämäläinen, R., and Reedijk, J. (1987) The Dinnuclear Unit $m$-Aqua-bis( $m$-carboxylato)dimetal. X-ray Structure and Magnetism of Cobalt and Nickel(II) Compounds Containing Bridging Carboxylato Groups and a Bridging Water Molecule, Polyhedron6, 1603.

55 Chaudhuri, P., Ouerbach, J., Wieghardt, K., Nuber, B., and Weiss, J. (1990) Synthesis, Electrochemistry, and Magnetic Properties of Binuclear Cobalt Complexes Containing the $\mathrm{Co}_{2}(m-\mathrm{X})(\mathrm{m} \text {-carboxylato })_{2}{ }^{+n}$ Core $(\mathrm{X}=$ $\mathrm{OH}, \mathrm{Cl}$, or $\mathrm{Br} ; n=1-3)$, J. Chem. Soc., Dalton Trans., 271.

56 Glerup, J., Goodson, P. A., Hodgson, D. J., and Michelsen, K. (1995) Magnetic Exchange through Oxalate Bridges: Synthesis and Characterization of (m-Oxalato)dimetal(II) Complexes of Manganese, Iron, Cobalt, Nickel, Copper, and Zinc, Inorg. Chem.34, 6255.

57 Kalinnikov, V. T., Rakitin, Y. V., and Hatfield, W. E. (1978) Magnetic Properties of Dimeric Cobalt(II) Carboxylates, Inorg. Chim. Acta31, 1.

58 Little, I. R., Straughan, B. P., and Thornton, P. (1986) Magnetic and Spectroscopic Study of Some Binuclear Ans Trinuclear Cobalt(II) Carboxylate Complexes, J. Chem. Soc., Dalton. Trans., 2211. 
59 Chevrier, B., D'Orchymont, H., Schalk, C., Tarnus, C., and Moras, D. (1996) The Structure of the Aeromonas proteolytica Aminopeptidase Complexed with a Hydroxamate Inhibitor. Involvement in Catalysis of Glu151 and Two Zinc lons of the Cocatalytic Unit, Eur. J. Biochem.237, 393.

60 Ustynyuk, L., Bennett, B., Edwards, T., and Holz, R. C. (1999) Inhibition of the Aminopeptidase from Aeromonas proteolytica by Aliphatic Alcholols. Characterization of the Hydrophobic Substrate Recognition Site, Biochemistry38, 11433.

61 Bienvenue, D., Bennett, B., and Holz, R. C. (2000) Inhibition of the Aminopeptidase from Aeromonas proteolytica by I-Leucinethiol: Kinetic and Spectroscopic Characterization of a Slow, Tight-Binding Inhibitor-Enzyme Complex, J. Inorg. Biochem.78, 43.

62 Bienvenue, D., Gilner, D., and Holz, R. C. (2002) Hydrolysis of Thiopeptides by the Aminopeptidase from Aeromonas proteolytica, Biochemistry41, 3712.

63 Bienvenue, D. L., Mathew, R. S., Ringe, D., and Holz, R. C. (2002) The Aminopeptidase from Aeromonas proteolytica Can Function as an Esterase, J. Biol. Inorg. Chem.7, 129.

1 Abbreviations: Hepes, 4-(2-hydroxyethyl)-1-piperazineethanesulfonic acid; Tricine, $N$ tris[hydroxymethyl]methylglycine; bestatin, [(2S,3R)-3-amino-2-hydroxy-4-phenylbutanoy]-leucine; LPA, I-leucine phosphonic acid; BuBA, 1-butaneboronic acid; AAP, aminopeptidase from Aeromonas proteolytica; bILAP, bovine lens leucine aminopeptidase; o-phen, 1,10-phenanthroline; EPR, electron paramagnetic resonance. 